\title{
Intercoder-Reliabilität bei der Berufscodierung nach der ISCO-88 und Validität des sozioökonomischen Status
}

\author{
Kai Maaz • Ulrich Trautwein • Cornelia Gresch • Oliver Lüdtke • \\ Rainer Watermann
}

\begin{abstract}
Zusammenfassung: Indikatoren der sozialen Herkunft gehören seit jeher zu den Standardinstrumenten der empirischen Bildungsforschung. Die Bildung valider Rangskalen und Kategoriensysteme zur Erfassung der sozialen Herkunft setzt eine differenzierte Erfassung des Berufs und der ausgeübten beruflichen Tätigkeit der Eltern voraus. Die Codierung dieser Angaben nach standardisierten Vorgaben ist ein komplexer Prozess. Der vorliegende Beitrag untersucht die IntercoderReliabilität von Berufscodierungen nach der ISCO-88 sowie die aus diesen Codes generierten Werte für den sozioökonomischen Status (ISEI) anhand der Angaben einer Zufallsstichprobe von 300 Abiturienten zum Beruf der Mutter und des Vaters. Hierfür wurde zum einen eine Doppelcodierung professioneller Codierer und zum anderen eine in Eigenregie durchgeführte Doppelcodierung vorgenommen. Die Ergebnisse zeigen, dass der ISCO-Code zwischen verschiedenen Codierungen nur in etwas mehr als 50 Prozent genau übereinstimmt. Die Validität des darauf beruhenden Indexes für den sozioökonomischen Status kann dagegen als sehr gut bezeichnet werden. Die Korrelationen zwischen den ISEI-Werten, die auf der Basis der Codierungen der unterschiedlichen Codierer generiert wurden, fielen sehr hoch aus. Die Vorhersagekraft des familiären Hintergrunds variierte nicht zwischen den Codierungen der unterschiedlichen Codierer.
\end{abstract}

Schlüsselwörter: Soziale Herkunft · Soziale Ungleichheit · Berufscodierung ·

Sozioökonomischer Status · Schulleistungsstudien · ISCO

\section{Intercoder-reliability for coding occupations according to ISCO-88 and the validity of socio-economic status}

\begin{abstract}
Indicators of social background belong to the standard set of instruments for empirical research in education. Construction of valid ranking scales and category systems for social background depends on a differentiated surveying of the occupation and vocational activity of parents. Coding such details using standard procedures is a complex process. This contribution investigates the intercoder-reliability of occupational codes according to ISCO-88 and the indicators for socio-economic status (ISEI) based on these codes using a random sample of 300 graduates surveyed on the occupation of their father and mother. To this aim, we compared a double-coding by professional coders and a double-coding by the research team. The results show a match of around 50 percent between the two coding groups. The validity of the index of socio-economic
\end{abstract}

(C) VS-Verlag 2009

Dr. habil. K. Maaz $(\bowtie) \cdot$ Univ.-Prof. Dr. U. Trautwein · Dipl.-Soz. Wiss. C. Gresch • Univ.-Prof. Dr. O. Lüdtke · Univ.-Prof. Dr. R. Watermann (Universität Göttingen) Max-Planck-Institut für Bildungsforschung, Lentzeallee 94, 14195 Berlin, Deutschland E-Mail: maaz@mpib-berlin.mpg.de 
status based on the data was, however, very good. The correlation between ISEI-values based on the coding from the different coders was very high. The predication of family background did not vary between the coding of the different coding groups.

Keywords: Coding occupations - ISCO $\cdot$ School achievement studies $\cdot$ Social background · Social inequality $\cdot$ Socio-economic status

\section{Einleitung}

In der empirischen Bildungsforschung stellen Angaben zum sozioökonomischen Hintergrund der Schülerinnen und Schüler bzw. des Schulumfeldes zentrale Analysevariablen dar. Insbesondere die Analyse sozialer Disparitäten der Bildungsbeteiligung und des Kompetenzerwerbs setzt die differenzierte und valide Erfassung von Merkmalen des sozialen Hintergrunds voraus. Alle großen Schulleistungsstudien haben sich diesem Thema gewidmet und greifen auf Indices zurück, die im Wesentlichen auf Angaben zum Beruf und zur beruflichen Tätigkeit der Eltern beruhen. Die zentrale Bedeutung dieser Variablen ist in jüngster Zeit besonders eindrücklich anhand der Befunde der PISA-Studie (Baumert, Stanat \& Watermann 2006) deutlich geworden.

Bei der Verwendung des sozialen Hintergrunds in empirischen Studien darf nicht übersehen werden, dass die Erhebung und Codierung dieser Merkmale mit Messproblemen behaftet sein kann. Eine erste Herausforderung besteht in der Erfassung der Hintergrundmerkmale. In Schulleistungsuntersuchungen werden Merkmale der sozialen Herkunft entweder über Informationen von Schülerinnen und Schülern gewonnen oder über einen Elternfragebogen von den Eltern direkt erfragt. Stammen die Informationen zu den Bildungs-, Ausbildungs- und Berufsmerkmalen von den Schülerinnen und Schülern, liegen sogenannte Proxy-Angaben zum sozialen Hintergrund vor. Die Validität dieser Informationen hängt so im Wesentlichen vom Wissen der Schülerinnen und Schüler über die Bildungs- und Berufsmerkmale ihrer Eltern ab. Systematische Analysen zur Übereinstimmung von Schüler- und Elternangaben liegen vor allem aus dem angloamerikanischen Raum vor (u. a. Lien, Friestad \& Klepp 2001); diese Studien kommen im Wesentlichen zu positiven Bewertungen dieser Übereinstimmung. Im deutschsprachigen Raum lassen sich vergleichsweise wenige Arbeiten zum diesem Thema finden (Bauer, Langenheim \& Schork 1984; Meulemann \& Wieken-Mayser 1984). In neueren Studien, bei denen auf Angaben von 15-Jährigen bzw. Abiturienten und ihren Eltern zurückgegriffen wurde, ließen sich insgesamt gute Übereinstimmungen in den zentralen Merkmalen des sozialen Hintergrunds feststellen (Kreuter, Maaz \& Watermann 2005, 2006; Maaz, Kreuter \& Watermann 2006; Maaz \& Watermann 2004).

Eine zweite wichtige Herausforderung besteht in dem aufwendigen Codierungsprozess, der insbesondere bei Berufsangaben notwendig wird. Bevor Angaben zum Beruf der Eltern in empirische Analysen eingehen, werden sie in den meisten Studien auf der Basis eines Codierschemas (i.d.R: ISCO=International Standard Classification of Occupation) durch die beteiligten Wissenschaftler oder durch spezialisierte Codierer in Kategorien überführt, aus denen dann wiederum Maße für das „Prestige“ bzw. den 
sozioökonomischen Status gebildet werden. Über die Reliabilität des Codierprozesses ist bislang jedoch wenig bekannt.

In der vorliegenden Studie haben wir deshalb für den familiären Hintergrund von 300 Abiturienten von zwei professionellen Instituten sowie zwei von uns selbst geschulten studentischen Hilfskräften Berufscodierungen vornehmen lassen. Daraufhin untersuchten wir systematisch, wie stark die Übereinstimmung der Berufscodes zwischen den vier Codierern ausfiel. Zudem überprüften wir, inwieweit sich Abweichungen in der Berufscodierung auf die daraus gebildeten Variablen zum sozioökonomischen Status auswirkten und welche Konsequenzen dies für die Vorhersagekraft dieser Indikatoren hatte.

\section{Erfassung der sozioökonomischen Stellung in der empirischen Bildungsforschung}

Die soziale Herkunft von Schülerinnen und Schülern wird üblicherweise mithilfe der sozioökonomischen Stellung ihrer Familien bestimmt, das heißt mithilfe von Daten zur relativen Position ihrer Eltern in einer sozialen Hierarchie, deren Ordnungsprinzipien in der Verfügung über finanzielle Mittel, Macht oder Prestige bestehen. Da Informationen über Einkommensverhältnisse, Macht und soziale Anerkennung von Individuen nicht einfach zu erhalten sind, wird die sozioökonomische Stellung in aller Regel über die berufliche Tätigkeit erfasst, die Hinweise auf jeden der drei Aspekte geben kann. Für die Beschreibung des sozioökonomischen Status aufgrund der beruflichen Tätigkeit kann dabei auf verschiedene methodische Systematisierungen (Codiersysteme, Indices und Kategoriensysteme) zurückgegriffen werden.

\subsection{Codiersysteme für Berufsangaben}

Für Deutschland können zwei Berufsklassifikationsschemata unterschieden werden: die speziell für Deutschland entwickelte Klassifizierung der Berufe (KldB-92), die mit über 2.000 Berufsklassen eine sehr differenzierte Einordnung der verschiedenen Berufe erlaubt, gut dokumentiert ist und beispielsweise vom Statistischen Bundesamt im Mikrozensus umgesetzt wird, und die International Standard Classification of Occupations (ISCO). Für die Umsetzung der ISCO kann auf Vorarbeiten zur internationalen Berufsklassifikation des Internationalen Arbeitsamts zurückgegriffen werden, das bereits 1958 und 1968 eine internationale Standardklassifikation der Berufe herausgegeben hat (vgl. ILO 1969), die 1988 an die Veränderungen der Arbeitswelt in den Industrienationen angepasst wurde (ISCO-88; siehe ILO 1990). ${ }^{1}$

ISCO und KldB basieren auf unterschiedlichen Logiken, die keine fehlerfreie Übersetzung von der einen in die andere Codierung zulassen. Während die ISCO auf der beruflichen Tätigkeit basiert, greift die KldB auf Berufsbezeichnungen zurück, die dann zu Klassen zusammengefasst werden. Eine genaue Übertragung der Codes zwischen diesen Codiersystemen ist somit nicht durchführbar, wenngleich entsprechende Umsteigeschlüssel auch für diesen Schritt von einigen Instituten angeboten werden. Geis und Hoffmeyer-Zlotnik (2001) konnten zeigen, dass bei einer automatischen Umsetzung von KldB-92 auf ISCO-88 die Fehlerrate beträchtlich ist. Etwa ein Drittel der Fälle in einem 
konkreten Datensatz weisen eine nicht ausschließliche (d.h. keine „eins zu eins genaue“) Codezuordnung auf. Aufgrund der internationalen Anwendungsmöglichkeit basieren die meisten Routinen zur Erstellung von Prestigemaßen oder dem sozioökonomischen Status auf Grundlage der ISCO-Klassifikation, die im Folgenden vorgestellt wird.

Ziel der ISCO-88 ist die Gliederung und das Zusammenfassen möglichst ähnlicher Berufe. Hierbei werden ca. 30.000 Berufe in 390 Kategorien für berufliche Tätigkeiten abgebildet. Die ISCO-88 legt vier Gliederungsebenen fest, bestehend aus zehn Berufshauptgruppen (inkl. Soldaten), 28 Berufsgruppen (Unterteilungen der Berufshauptgruppen), 116 Berufsuntergruppen (Unterteilungen der Berufsgruppen) und 390 Berufsgattungen (Unterteilungen der Berufsuntergruppen). Hauptkriterien für die Gliederung und das Zusammenfassen der Berufe sind das Skill Konzept und das Konzept der beruflichen Tätigkeit. Das Skill Konzept lässt sich in die Dimensionen „Skill Level“ und „Skill Specialisation“ unterteilen, die zur Gliederung auf der Ebene der Berufshauptgruppen (ISCO-1-Steller) eingesetzt werden. Skill Level ordnen die Berufe auf der obersten Ebene der Berufshauptgruppe quasi-hierarchisch. Sie beschreiben die Qualifikationsanforderungen, die an Berufe gestellt werden, und verkörpern so kein personengebundenes Merkmal, sondern ein berufs- bzw. arbeitsplatzbezogenes Kriterium. Die ISCO-88 unterscheidet vier relativ grobe Skill Level-Kategorien, die in Anlehnung an die International Standard Classification of Education (ISCED) operationalisiert werden (vgl. Abb. 1). Der erste Skill Level subsumiert die Ausbildung im Primarbereich (Grundschule). Der zweite Skill Level fasst die Ausbildung im Sekundarbereich I und II zusammen. Neben formalen Qualifikationen kann im zweiten Skill Level zusätzlich eine betriebliche Ausbildung und/oder Berufserfahrung erforderlich sein. Der Skill Level 3 umfasst in der Regel die im Alter von 17 oder 18 Jahren beginnende Ausbildung im postsekundären bzw. auch im tertiären Bereich mit einer Dauer von ca. vier Jahren (z. B. Berufsakademien, Fachhochschulen). Der vierte Skill Level fasst alle Ausbildungen zusammen, die zu einem Universitätsabschluss, einem Postgraduiertenabschluss oder einem gleichwertigen Abschluss führen (ILO 1990; Elias \& Birch 1993; Wallner-Paschon 2004). Anforderungen, die mit dem Konzept der Skill Level spezifiziert werden, müssen nicht zwingend durch formale Bildung und den damit korrespondierenden Zertifikaten assoziiert sein. Die erforderlichen Anforderungen können auch durch informelles Training oder Berufserfahrung erworben werden. Mit der Skill Specialisation werden Gebiete unterschieden, auf denen bestimmte Kenntnisse erforderlich sind, um die Aufgaben kompetent bewältigen zu können. Damit wird die Art der ausgeübten Tätigkeit näher bestimmt, indem z. B. auf spezifische Merkmale, wie verwendete Maschinen und Materialien, sowie auf Arten von produzierten Gütern und Dienstleistungen Bezug genommen wird.

Skill Level und Skill Specialisation führen zur Gliederung der Berufe auf der Ebene der Berufshauptgruppe. In Abb. 1 ist diese Gliederung dargestellt. Dabei ist zu erkennen, dass den Berufshauptgruppen 0 und 1 kein Skill Level zugeordnet ist. In diesen Berufshauptgruppen werden Aspekte der beruflichen Tätigkeit, wie Leitungsfunktionen im politischen und wirtschaftlichen Bereich bzw. militärische Aufgaben, als wichtigere Abgrenzungskriterien herangezogen, die sich nicht in das Konzept der Skill Level überführen lassen bzw. nicht darauf beruhen. Innerhalb dieser Berufshauptgruppen erfolgt mithilfe der Skill Specialisation eine weitere Untergliederung in insgesamt 28 Berufsgruppen. Nach dem Konzept der Skill Specialisation werden die Berufsgruppen in 116 


\begin{tabular}{|c|c|c|c|}
\hline Code & Berufshauptgruppen & $\begin{array}{l}\text { Skill } \\
\text { Level }\end{array}$ & Skill Level Beschreibung \\
\hline 1 & $\begin{array}{l}\text { Angehörige gesetzgebender } \\
\text { Körperschaften, leitende } \\
\text { Verwaltungsbedienstete und } \\
\text { Führungskräfte in der Privatwirtschaft }\end{array}$ & - & \\
\hline 2 & Wissenschaftler & 4 & $\begin{array}{l}\text { Gliederungskategorien } 6 \text { und } 7 \text { der } \\
\text { ISCED, umfasst die im Alter von } 17 \text { oder } \\
18 \text { Jahren beginnende Ausbildung mit } \\
\text { einer Dauer von drei, vier oder mehr } \\
\text { Jahren, die zu einem Universi- } \\
\text { tätsabschluss, einem } \\
\text { Postgraduiertenabschluss oder einem } \\
\text { gleichwertigen Abschluss führt. }\end{array}$ \\
\hline 3 & $\begin{array}{l}\text { Techniker und gleichrangige } \\
\text { nichttechnische Berufe }\end{array}$ & 3 & $\begin{array}{l}\text { Gliederungskategorie } 5 \text { der ISCED, } \\
\text { umfasst die im Alter von } 17 \text { oder } 18 \\
\text { Jahren beginnende Ausbildung im } \\
\text { postsekundären bzw. auch im tertiären } \\
\text { Bereich mit einer Dauer von ca. vier Jahren } \\
\text { (z. B. Berufsakademien, } \\
\text { Fachhochschulen). }\end{array}$ \\
\hline 4 & $\begin{array}{l}\text { Bürokräfte, kaufmännische } \\
\text { Angestellte }\end{array}$ & 2 & \multirow{5}{*}{$\begin{array}{l}\text { Gliederungskategorien } 2 \text { und } 3 \text { der } \\
\text { ISCED, erste und zweite Stufe der } \\
\text { Sekundarausbildung. Stufe } 1 \text { beginnt im } \\
\text { Alter von } 11 \text { oder } 12 \text { Jahren und dauert ca. } \\
\text { drei Jahre, Stufe } 2 \text { beginnt im Alter von } \\
14 \text { oder } 15 \text { Jahren und dauert ca. drei } \\
\text { Jahre. Eine betriebliche Ausbildung } \\
\text { und/oder Berufserfahrung kann } \\
\text { erforderlich sein. }\end{array}$} \\
\hline 5 & $\begin{array}{l}\text { Dienstleistungsberufe, Verkäufer in } \\
\text { Geschäften und auf Märkten }\end{array}$ & 2 & \\
\hline 6 & $\begin{array}{l}\text { Fachkräfte in der Landwirtschaft und } \\
\text { Fischerei }\end{array}$ & 2 & \\
\hline 7 & Handwerks- und verwandte Berufe & 2 & \\
\hline 8 & $\begin{array}{l}\text { Anlagen und Maschinenbediener } \\
\text { sowie Montierer }\end{array}$ & 2 & \\
\hline 9 & Hilfsarbeitskräfte & 1 & $\begin{array}{l}\text { Gliederungskategorie } 1 \text { der ISCED, die } \\
\text { Primarausbildung, die üblicherweise im } \\
\text { Alter von } 5 \text { bis } 7 \text { Jahren beginnt und etwa } \\
5 \text { Jahre dauert. }\end{array}$ \\
\hline 0 & Soldaten & - & \\
\hline
\end{tabular}

Quelle: ILO 1990, S. 2 f.; Elias \& Birch 1993, S. 10; Wallner-Paschon 2004, S. 164.

Abb. 1: Berufshauptgruppen der ISCO-88 mit den zugehörigen Skill Levels und ISCED-Kategorien sowie deren Beschreibungen

Berufsuntergruppen untergliedert, die sich schließlich nach dem Konzept der beruflichen Tätigkeit in 390 Berufsgattungen ausdifferenzieren. Das Konzept der beruflichen Tätigkeit definiert die von einer Person wahrzunehmenden Aufgaben und Pflichten. Nach der ISCO ist die berufliche Tätigkeit auf der untersten Ebene angeordnet und stellt die zu klassifizierende Einheit dar. Da sich Tätigkeiten in ihren Aufgaben und Pflichten ähneln, werden sie zu einzelnen Berufen (Berufsgattungen) zusammengefasst. Dabei kann es sein, dass sich die einzelnen Tätigkeiten innerhalb einer Berufsgattung in Bezug auf die 
zu erbringenden Leistungen (output) voneinander unterscheiden. Trotz dieser Variabilität sind die auszuführenden Tätigkeiten hinreichend ähnlich, insbesondere in Bezug auf Fähigkeiten, die für deren Ausübung benötigt werden (input).

Die Erfassung der beruflichen Tätigkeit erfolgt im Fragebogenformat über die Abfrage des derzeitig ausgeübten Berufs und der konkreten Tätigkeit, der in diesem Beruf nachgegangen wird. Diese differenzierte Erfassung führt zu der Annahme, dass unabhängige Codierungen in den meisten Fällen zum gleichen Ergebnis führen. Allerdings lassen die Angaben in vielen Fällen mehrere Berufscodes zu. So ist es z. B. bei nicht ganz genauer Textangabe schwierig zu unterscheiden, ob eine Person als Kassierer in einer Bank (4211) oder als Bankschalterangestellter (4212) arbeitet. Für die Codierung schwieriger oder nicht eindeutiger Berufsangaben können Codierer auf zusätzliche Informationen (z. B. berufliche Stellung) zurückgreifen, um eine zuverlässige Codierung zu gewährleisten.

Bislang liegen so gut wie keine Studien vor, die sich mit der Analyse der IntercoderReliabilität von Berufscodierungen beschäftigen. Hoffmeyer-Zlotnik, Hess und Geis (2006) konnten im Rahmen der Vorstellung eines Instrumentes zur computergestützten Codierung nach der ISCO-88 zeigen, dass im Feld vorgenommene Codierungen in ca. zwei Dritteln aller Fälle mit einer nachträglichen Codierung durch professionelle Codierer übereinstimmten. In einer weiteren bekannten Studie wurden Reliabilitätsanalysen der Berufscodierungen auf Basis der PISA-2003-Schülerdaten für Österreich vorgenommen. Diese Studie konnte zeigen, dass sich keine perfekte Übereinstimmung zwischen unterschiedlichen Codierern ermitteln ließ. Die Maße der prozentualen Übereinstimmung für die Doppelcodierung der Berufe der Eltern schwanken zwischen ca. 70 Prozent für Berufsangaben der Mütter und 55 Prozent bei den Vätern (Wallner-Paschon 2004).

\subsection{Index für den sozioökonomischen Status}

Auf der Basis der ISCO-Codes lassen sich Rangskalen und Kategoriensysteme bilden, mit deren Hilfe die Stellung von Personen in der sozialen Hierarchie einer Gesellschaft bestimmt werden kann (vgl. Baumert/Maaz 2006). Für die Messung des sozioökonomischen Status wird in vielen Studien der von Ganzeboom und Mitarbeitern entwickelte International Socio-Economic Index of Occupational Status (ISEI) herangezogen (vgl. Ganzeboom, de Graaf \& Treiman 1992, S. 10 f.). Der ISEI besitzt den Vorzug, die ökonomische Stellung vom Berufsprestige zu trennen. Die Autoren gehen davon aus, dass Schul- und Berufsbildung über Berufe in Einkommen und in Chancen zur Teilhabe an Macht umgesetzt werden; da Berufe bestimmte Qualifikationen voraussetzen und zu bestimmten Einkommen führen, sind sie als Vermittler zwischen Bildungsabschlüssen und Einkommenslagen zu betrachten. Demgemäß ordnen die Autoren Berufe so auf einer linearen Skala an, dass in einem pfadanalytischen Modell der indirekte Einfluss der Bildung auf das Einkommen maximiert und der direkte Einfluss gleichzeitig minimiert wird (vgl. ebd.). Der Internationale Sozioökonomische Index, der auf der Basis von Daten zu Bildung, Beruf und Einkommen von 74.000 Beschäftigten aus 16 Ländern auf diese Weise erzeugt worden ist, hat sich in Vergleichsuntersuchungen bewährt. Er wurde beispielsweise in PISA 2000 bei allen internationalen Vergleichen als Standardindikator verwendet. 


\section{Fragestellung}

Angesichts des anspruchsvollen Codierprozesses, der bei Berufscodierungen notwendig wird, ist anzunehmen, dass keine perfekte Reliabilität erreicht werden kann (vgl. Wallner-Paschon 2004). In diesem Beitrag untersuchen wir deshalb, wie reliabel Berufscodierungen, wie sie typischerweise in Schulleistungsstudien verwendet werden, sind. Zudem untersuchen wir, welche Auswirkungen Einschränkungen in der Reliabilität für substanzwissenschaftliche Fragestellungen haben könnten.

$\mathrm{Zu}$ diesem Zweck wurden zwei Institute, die eine Codierung von Berufen nach der ISCO-88 bereits häufig und auch im Rahmen von bedeutenden Schulleistungsstudien durchgeführt haben, unabhängig voneinander und unter Verwendung der identischen Informationen mit einer Berufscodierung nach der ISCO- 88 beauftragt. Zusätzlich wurde eine Doppelcodierung derselben Berufe von zwei geschulten studentischen Mitarbeitern des Forschungsprojektes vorgenommen. Damit lässt sich prüfen, wie hoch die Übereinstimmung zwischen zwei unerfahrenen Codierern sowie zwischen „professionellen“ Codierungen und unerfahrenen Codierern ausfällt. Vor dem Hintergrund, dass gerade in kleineren Forschungsprojekten die Codierung der Berufe oft nicht an professionelle Institute abgegeben wird, besitzt dieser Analyseschritt eine große praktische Relevanz.

Im Einzelnen prüften wir folgende Hypothesen: Erstens wurde davon ausgegangen, dass die Übereinstimmung zwischen den unterschiedlichen Codierungen mit zunehmendem Differenzierungsgrad der Gliederungsebenen abnimmt. Dabei sollten sich insgesamt etwas niedrigere Übereinstimmungskoeffizienten für die unerfahreneren Codierer ergeben.

Zweitens prüften wir, ob die Anzahl der zusätzlich zu den Berufsangaben herangezogenen Informationen einen Effekt auf die Übereinstimmung unterschiedlicher Codierungen aufweist. Wir vermuteten, dass die Übereinstimmung dann am höchstens sein sollte, wenn allein auf Basis der Berufsangaben eine eindeutige Codierung möglich ist und keine weiteren Informationen benötigt werden.

In einem dritten Schritt untersuchten wir, wie stark Aussagen zum sozioökonomischen Status von einer etwaigen Unreliabilität der Codierung betroffen sein würden. Da der ISCO-Code für die meisten Fragestellungen der Bildungsforschung nicht in die Analyse eingeht, sondern die Grundlage für die Bildung valider Skalen oder Kategoriensysteme ist, wurde hierfür der aus dem ISCO-Code generierte Index für den sozioökonomischen Status (ISEI) herangezogen. Der ISEI-Index eignet sich besonders, weil seine Werte direkt und ohne zusätzliche Transformationen sowie ohne Berücksichtigung weiterer Indikatoren aus den ISCO-Codes gebildet werden. Im Hinblick auf die Mittelwerte und Standardabweichungen des ISEI gingen wir davon aus, dass sich diese zwischen den professionellen Codierungen nicht voneinander unterscheiden. In Bezug auf die eigenständige Codierung durch unsere studentischen Mitarbeiter hatten wir keine Hypothese.

Die abschließenden Analysen galten dem Zusammenhang zwischen den generierten ISEI-Werten und zentralen Drittvariablen wie Leistungsmerkmalen und Angaben zum kulturellen und sozialen Kapital. Hierfür wurde erstens geprüft, ob es Unterschiede in der Höhe der Korrelationskoeffizienten gibt, wenn die Codierungen unterschiedlicher Codierer verwendet werden. Zum anderen wurde auch ein Regressionsmodell spezifiziert, in dem die prädiktive Wirkung unterschiedlicher Codierungen überprüft wurde. Wir gingen 
dabei davon aus, dass sich sowohl hinsichtlich der Korrelationen als auch in der prädiktiven Wirkung keine statistisch signifikanten Unterschiede zwischen den Codierungen ermitteln lassen würden.

\section{Methode}

\subsection{Datengrundlage}

Die Datengrundlage für die nachfolgenden Analysen bildet eine Stichprobe von $N=300$ Abiturientinnen und Abiturienten der Längsschnittstudie „Transformation des Sekundarschulsystems und akademische Karrieren“" (TOSCA; Köller et al. 2004; Trautwein et al. 2007), die in Kooperation zwischen dem Berliner Max-Planck-Institut für Bildungsforschung (Forschungsbereich Erziehungswissenschaft und Bildungssysteme) und dem Institut zur Qualitätsentwicklung im Bildungswesen durchgeführt wird. Diese 300 Abiturienten wurden per Zufall aus der ursprünglichen Gruppe von 4.730 Abiturienten aus 149 allgemeinbildenden und beruflichen Gymnasien, die im Jahr 2002 im Bundesland Baden-Württemberg an der TOSCA-Studie teilnahmen, ausgewählt. Die in Schulleistungsstudien vorzufindende Mehrebenenstruktur, nach der Schülerinnen und Schüler innerhalb von Schulen oder Klassen geschachtelt sind, kann aufgrund der geringen Zahl von durchschnittlich rund zwei Schülern pro Schule in den nachfolgend berichteten Analysen vernachlässigt werden.

\subsection{Codierer}

Die Berufscodierung wurde von zwei professionellen und voneinander unabhängigen Instituten vorgenommen. Beide Institute bieten ISCO-Codierungen als kostenpflichtige Dienstleistungen an und weisen eine langjährige Erfahrung bezüglich der Berufscodierung auf. Den Instituten wurde bei Auftragstellung nicht mitgeteilt, dass es sich um Doppelcodierungen handelt, deren Übereinstimmung im Rahmen einer wissenschaftlichen Untersuchung untersucht werden würde.

Zusätzlich erfolgte eine ISCO-Codierung derselben Berufsangaben durch zwei geschulte studentische Mitarbeiter des Forschungsprojekts. Es handelte sich um einen Psychologiestudenten im Grundstudium und einen Studenten der Politikwissenschaften am Anfang des Hauptstudiums ohne jegliche Erfahrung im Umgang mit Berufsklassifikationsschemata oder der Berufsvercodung. Die Schulung wurde von einer Mitarbeiterin durchgeführt, die bereits Erfahrung in der Vercodung und den Umgang mit Klassifikationsschemata aufweist. Die Codierer wurden angewiesen, die Codierung der Berufe nach der ISCO-88 vorzunehmen und konnten hierfür das Handbuch für die Berufsvercodung (Geis 2007) vom Zentrum für Umfragen, Methoden und Analysen (ZUMA) und die auf den Webseiten von ZUMA ${ }^{2}$ erhältlichen Übersichtsinformationen nutzen. Weitere Informationen konnten sie bei Bedarf auch dem Handbuch der Klassifikation der Berufe entnehmen, das aber nicht Grundlage der Codierung war. Die Studenten führten die Codierung ohne zeitliche Begrenzung auf Basis der offenen Berufsbezeichnung und einer präzisierenden Berufsbeschreibung durch. Weiterhin konnten sie bei Bedarf auf den Schulabschluss, die Schuldauer, Ausbildungs- 
abschluss, berufliche Stellung und den Arbeitgeber (Öffentlicher Dienst, Selbstständigkeit oder Unternehmen) zurückgreifen (vgl. Hoffmeyer-Zlotnik, Hess \& Geis 2004). Die Studenten waren über das Forschungsziel der Studie informiert und wurden angehalten, sich nicht über spezielle Berufsvercodungen auszutauschen. Insgesamt lagen für jede Berufsangabe vier Codes nach der ISCO-88 vor, zwei von den professionellen Codierinstituten und zwei von den studentischen Mitarbeitern des Projektes.

\subsection{Instrumente}

\subsubsection{Berufstätigkeit und sozioökonomischer Status}

Die Erhebung der Berufstätigkeit der Eltern erfolgte in der TOSCA-Studie - getrennt für den Vater und die Mutter - über zwei offene Fragen. Zuerst wurde nach dem Beruf gefragt, den der Vater/die Mutter zurzeit ausüben, bzw. bei nicht erwerbstätigen Eltern(teilen) nach dem zuletzt ausgeübten Beruf. Anschließend wurde nach einer Beschreibung der konkreten beruflichen Tätigkeit gefragt, die von dem Vater/der Mutter in den jeweiligen Berufen ausgeübt werden bzw. wurden. Damit orientiert sich die Erfassung des Berufs bzw. der beruflichen Tätigkeit an den in den Demographischen Standards vorgeschlagenen Formulierungen. Lediglich auf den dritten dort aufgeführten Schritt, der Frage, ob dieser Beruf noch einen besonderen Namen hat (vgl. Statistisches Bundesamt 2004), wurde verzichtet. Diese differenzierte, zweischrittige Erfassung ist erforderlich, um der beruflichen Tätigkeit auch einen genauen Berufscode zuordnen zu können. Ein angestellter Handwerksmeister, der in der Funktion als Werkstattleiter hauptsächlich unternehmerischen Tätigkeiten nachgeht, bekommt demzufolge einen anderen Code als ein Handwerksmeister, der in einer Werkstatt arbeitet. Diese Angaben bildeten die primäre Grundlage, die die Codierer für die Codierung der Berufstätigkeit nach der ISCO-88 (ILO 1990) verwenden konnten. Zusätzlich zu diesen Angaben standen den Codierern weitere Informationen zur Verfügung. Im Wesentlichen konnte bei Bedarf auf Angaben zum Bildungshintergrund, zum Erwerbstätigkeitsstatus und zur beruflichen Stellung zurückgegriffen werden. Aus den ISCO-Codes wurde auf der Basis der entsprechenden Vorgaben durch die Autoren dieser Studie der international vergleichbare Index ISEI (Ganzeboom, de Graaf \& Treiman 1992) als Maß für den sozioökonomischen Status gebildet.

\subsubsection{Zusätzlich herangezogene Informationen der eigenen Codierung}

Anzahl zusätzlich herangezogener Informationen zur Codierung. Die beiden projektinternen Codierer sollten für jede Codierung angeben, wie viele Informationen sie für die Codierung herangezogen haben. Dabei wurden vier Kategorien unterschieden: (1) nur die Angaben zum Beruf, (2) die Angaben zum Beruf und eine weitere Information, (3) die Angaben zum Beruf und zwei weitere Informationen, (4) die Angaben zum Beruf und drei oder mehr weitere Informationen. Aus den Angaben beider Codierer wurde ein Maß mit drei Ausprägungen generiert: (1) Beide Codierer haben maximal eine Zusatzinformation benutzt, (2) ein Codierer hat maximal eine Zusatzinformation und ein Codierer hat mehrere Zusatzinformationen genutzt, (3) beide Codierer haben wenigstens zwei Zusatzinformationen genutzt. 
Subjektive Schwierigkeit der Codierung. Für jede Codierung sollten die studentischen Codierer auf einer siebenstufigen Skala einschätzen, wie sicher sie sich bei der Codierung waren, wobei ein Wert von 1 für 0 Prozent, ein Wert von 4 für 50 Prozent und ein Wert von 7 für 100 Prozent stand.

\subsubsection{Instrumente zur Überprüfung der Kriteriumsvalidität}

Die Abiturnoten der Schülerinnen und Schüler sowie deren Prüfungsleistungen wurden den offiziellen Schulakten entnommen. Mathematikleistungen wurden mit Originalitems (Oberstufentest) aus der Third International Mathematics and Science Study (TIMSS) erhoben (für Details vgl. Watermann, Nagy \& Köller 2004). Die Englischleistung wurde mit einer Kurzversion des „Test of English as a Foreign Language“ (TOEFL) erhoben, der vom Educational Testing Service (ETS) in Princeton (vgl. Educational Testing Service 1997) entwickelt wurde (für Details vgl. Köller \& Trautwein 2004). Die kognitiven Grundfähigkeiten von Abiturienten wurden mit zwei Untertests des Kognitiven Fähigkeitstests (KFT), die als Markertests für schlussfolgerndes Denken gelten, erhoben (Heller \& Perleth 2000). Als zentraler Indikator für den Besitz von Kulturgütern wurde der Bücherbesitz in der elterlichen Wohnung herangezogen. Hierbei werden vier Ausprägungen berücksichtigt: (1) keine Bücher, (2) 1-10 Bücher, (3) 11-50 Bücher, (4) 51-100 Bücher, (5) 101-250 Bücher, (6) 251-500 Bücher, (7) mehr als 500 Bücher. Die kulturelle Praxis subsumiert verschiedene kulturelle Tätigkeiten (z. B. Theaterbesuche), die mit einer vierstufigen Likert-Skala erfasst wurden (interne Konsistenz: Cronbachs $\alpha=0,66$ ). Die soziale Praxis misst Aktivitäten, die Eltern mit ihren Kindern zusammen ausüben (z.B. mit den Eltern über politische und soziale Fragen diskutieren) und wurde mit einer fünfstufigen Likert-Skala erfasst (interne Konsistenz: Cronbachs $\alpha=0,70$ ).

\section{4 Übereinstimmungsmaße}

Die strengste Annahme in Bezug auf die Übereinstimmung unterschiedlicher Berufscodierungen am Beispiel der ISCO-Codes besteht in der faktischen Übereinstimmung verschiedener Codierungen. Dazu wird häufig auf die prozentuale Übereinstimmung (PÜ) zurückgegriffen, die das einfachste Maß der Übereinstimmungsmessung symbolisiert (vgl. Wirtz \& Caspar 2002). Sie gibt den prozentualen Anteil der Fälle an, in denen unterschiedliche Codierungen zu demselben Berufscode führen. In einer Kontingenztabelle eines zweimalig gemessenen Merkmals (durch Codierung 1 und Codierung 2) wären die Fälle mit gleichen Werten in den Zellen der Hauptdiagonalen lokalisiert. Die Betrachtung einer direkten Übereinstimmung zwischen zwei unabhängigen Codierungen ist sehr anschaulich, berücksichtigt aber nicht, dass bereits per Zufall die Codes der Codierer übereinstimmen können. Mit der Berechnung von Cohens к (kappa) (vgl. Cohen 1960) kann diese Einschränkung berücksichtigt werden. Grundlage für die Berechnung von kappa ist die prozentuale Übereinstimmung. Zusätzlich wird das Verhältnis zwischen beobachteten und bei Zufall erwarteten Übereinstimmungen berücksichtigt. Kappa berechnet so den Anteil an Übereinstimmungen, der über die rein zufällig zu erwartende Übereinstimmung hinausgeht. Es ist ein standardisiertes Maß, das Werte zwischen 0 und 1 annehmen kann. Kappa nimmt den Wert 0 an, wenn die faktische Übereinstimmung derjenigen entspricht, 
die allein per Zufall zu erwarten ist; kappa wird 1 im Falle perfekter Übereinstimmung. Je größer die faktische Übereinstimmung, desto größer wird kappa für ein gegebenes Paar von Marginalverteilungen. Die Berechnung der Kappawerte erfolgte in der vorliegenden Studie mit dem Programm Stata. Stata ermöglicht es, kappa auch dann zu berechnen, wenn nicht alle Zellen in beiden betrachteten Variablen besetzt sind.

Bei der Untersuchung der Übereinstimmung verschiedener Berufscodierungen ist es weiterhin sinnvoll, zwischen faktischer und relativer Übereinstimmung zu unterscheiden (vgl. Agresti 1990). So kann die relative Übereinstimmung (bzw. Assoziation) zwischen zwei Merkmalen stark sein, nicht jedoch die faktische. Ein Beispiel verdeutlicht dies: Wären alle Angaben der Codierung 1 um eine Einheit größer als die korrespondierenden Angaben der Codierung 2, so läge zwar eine perfekte Assoziation bzw. Korrelation vor, jedoch keinerlei faktische Übereinstimmung dieser Paare. Zur Beschreibung der Assoziation von zwei Variablen existieren unterschiedliche Korrelationsmaße, die je nach Skalenniveau der Variablen benutzt werden können.

Von einer strukturellen Übereinstimmung soll in diesem Zusammenhang gesprochen werden, wenn die aus unterschiedlichen Codierungen generierten Indices zum sozioökonomischen Status in gleicher Weise mit zentralen Drittvariablen korreliert sind. Zum Beispiel kann gefragt werden, ob die Schätzung der Korrelation zwischen dem sozioökonomischen Status und der Mathematikleistung bei Verwendung von SES-Werten auf Grundlage der Codierung 1 auf der einen Seite und auf Grundlage der Codierung 2 auf der anderen Seite identisch ausfällt.

\section{Ergebnisse}

\section{1 Übereinstimmung der Berufscodierungen}

Betrachtet man zunächst bei den professionellen Codierungen die Berufshauptgruppe (ISCO-1-Steller) als geringste Differenzierungsebene der ISCO, lässt sich für die Berufstätigkeit des Vaters eine Übereinstimmung von 74,7 Prozent ermitteln $(\kappa=0,68)$. Ein Kappa-Wert von 0,68 deutet hier insgesamt auf eine gute Übereinstimmung der Codierungen hin, wenngleich berücksichtigt werden muss, dass es sich hier lediglich um den Einsteller der Codierung handelt. Allerdings gibt die Berufshauptgruppe nur ein sehr grobes Bild der beruflichen Tätigkeit wieder. So kann zwar zwischen verschiedenen Berufshauptgruppen, z. B. „Wissenschaftler“ und „Handwerks- und verwandte Berufe“, unterschieden werden, die bereits auf unterschiedliche Qualifikationsanforderungen hinweisen, eine differenzierte Beschreibung des Berufs sowie des sozioökonomischen Status oder des Berufsprestiges sind allerdings noch nicht möglich. Beispielsweise subsumiert die Berufshauptgruppe 2 „Wissenschaftler“ typische Wissenschaftsberufe, wie sie in den verschiedenen Naturwissenschaften vorzufinden sind (z. B. Physiker, Chemiker, Biologen), den Universitätsprofessor, aber auch angewandte Wissenschaftsberufe wie Ärzte, Lehrkräfte im Schulbetrieb und sonstige Wissenschaften sowie verwandte Berufe (Psychologen, Soziologen, Sozialarbeiter). Eine weitere Differenzierung in Berufsgruppen, Berufsuntergruppen und Berufsgattungen ist demnach erforderlich. Für den Beruf des Vaters sank die Übereinstimmung hierbei bis auf 47,8 Prozent $(\kappa=0,47)$ auf der Ebene 
der Berufsgattungen, also dem konkreten vierstelligen ISCO-Code (vgl. Tab. 1). Ein vergleichbares Muster konnte auch für die Berufe der Mutter ermittelt werden. Die Übereinstimmung des vierstelligen ISCO-Codes lag bei den Müttern mit 52 Prozent $(\kappa=0,51)$ geringfügig über dem Wert, der für die Väter ermittelt wurde (vgl. Tab. 1). Die Übereinstimmungswerte von ca. 50 Prozent erscheinen auf den ersten Blick sehr gering. Immerhin stimmt jede zweite Codierung zwischen den beiden Instituten nicht überein. Diese geringe Übereinstimmung resultiert aus einer strengen Regel für die Berechnung der Übereinstimmung. Als Kriterium der Übereinstimmung auf Ebene der Berufsgattungen wurde der konkrete vierstellige ISCO-Code zugrunde gelegt, der mitunter verschiedene Angaben zulässt. Des Weiteren können die Abweichungen zwischen zwei Codierungen inhaltlich so gering sein, dass sie praktisch vernachlässigt werden können.

Für die eigenständige Codierung der Berufsangaben durch geschulte studentische Mitarbeiter erbrachte die Doppelcodierung vergleichbare Übereinstimmungen, wie sie zwischen den professionellen Codierungen ermittelt wurden. Die Berufshauptgruppen der Berufe der Väter stimmten in 74,7 Prozent aller Codierungen überein $(\kappa=0,68)$ und die der Mütter in 73,4 Prozent der Codierungen $(\kappa=0,66)$. Für den vierstelligen ISCO-Code (die Berufsgattung) der beiden Codierer konnte bei den Vätern eine Übereinstimmung von 49,8 Prozent $(\kappa=0,49)$ und bei den Mütter von 47,1 Prozent $(\kappa=0,46)$ ermittelt werden (vgl. Tab. 1).

Die Übereinstimmungen der eigenen Codierungen mit denen der professionellen Codierungen fallen insgesamt nur geringfügig geringer aus als die der professionellen Codierungen. Bei den Mutter-Angaben schwankte die prozentuale Übereinstimmung der vierstelligen ISCO-Codes je nach Vergleich zwischen 41 und 56 Prozent und bei den Vätern zwischen 41 und 47 Prozent (vgl. Tab. 1).

Insgesamt bestätigte sich also die Annahme, dass keine perfekte Übereinstimmung zwischen verschiedenen Codierungen ermittelt werden konnte. Die Übereinstimmung nahm mit zunehmendem Differenzierungsgrad der Gliederungsebenen der ISCO stetig ab. Etwas überraschend fielen die Übereinstimmungen der unerfahrenen Codierer aus. Diese erreichten Übereinstimmungskoeffizienten, die mit denen der professionellen Codierungen vergleichbar waren. Zwischen den unerfahrenen und den professionellen Codierungen fielen die Übereinstimmungsmaße wie erwartet etwas geringer aus. Inwieweit die Übereinstimmung auf Ebene der Berufsgattung als hinreichend gut bezeichnet werden kann, hängt im Wesentlichen von dem Kriterium der Übereinstimmung ab.

Betrachtet man die Übereinstimmung der verschiedenen Codierungen in Abhängigkeit von der Anzahl der zusätzlich verwendeten Informationen, zeigte sich folgendes Bild (tabellarisch nicht ausgewiesen): Auf die Übereinstimmung der Angaben zum Beruf der Mutter hatten zusätzlich verwendete Informationen keinen Einfluss. Der Verzicht bzw. die Hinzunahme von zusätzlichen Informationen verbesserte die Übereinstimmung nicht, verschlechterte sie aber auch nicht. Für die Väter konnte hingegen ein Zusammenhang ermittelt werden. Wurden für die Codierung neben den Berufsangaben keine weiteren Informationen zugezogen, fiel der Mismatch immer größer aus als die Übereinstimmung. Noch deutlicher wird der Mismatch für den Fall, dass beide Codierer viele Zusatzinformationen zur Codierung genutzt haben. Die Hinzunahme möglichst vieler Zusatzinformationen erhöht demnach nicht die Übereinstimmung zwischen verschiedenen Codierungen. Dieser Befund deutet darauf hin, dass Zusatzinformationen ausschließlich 
Tab. 1: Übereinstimmung der ISCO-Codes auf Basis von professionellen Codierungen und Eigencodierungen, differenziert nach Ebenen der Codierung (prozentuale Übereinstimmung und kappa)

\begin{tabular}{|c|c|c|c|c|c|c|c|c|c|c|c|c|}
\hline \multicolumn{13}{|c|}{ Für Angaben zum Beruf der Mutter } \\
\hline \multirow[b]{2}{*}{ ISCO-Codierung } & \multicolumn{2}{|c|}{ Vgl_1 } & \multicolumn{2}{|c|}{ Vgl_2 } & \multicolumn{2}{|c|}{ Vgl_3 } & \multicolumn{2}{|c|}{ Vgl_4 } & \multicolumn{2}{|c|}{ Vgl_5 } & \multicolumn{2}{|c|}{ Vgl_6 } \\
\hline & $\overline{\mathrm{PÜ}}$ & $\kappa$ & PÜ & $\kappa$ & PÜ & $\kappa$ & PÜ & $\kappa$ & PÜ & $\kappa$ & PÜ & $\kappa$ \\
\hline $\begin{array}{l}\text { Berufshauptgruppe } \\
\text { (Einsteller) }\end{array}$ & 74,1 & 0,67 & 73,1 & 0,66 & 67,5 & 0,59 & 69,9 & 0,61 & 71,9 & 0,64 & 73,4 & 0,66 \\
\hline $\begin{array}{l}\text { Berufsgruppe } \\
\text { (Zweisteller) }\end{array}$ & 63,8 & 0,60 & 68,5 & 0,65 & 60,3 & 0,56 & 61,4 & 0,58 & 61,8 & 0,58 & 63,8 & 0,60 \\
\hline $\begin{array}{l}\text { Berufsuntergruppe } \\
\text { (Dreisteller) }\end{array}$ & 56,0 & 0,54 & 60,1 & 0,59 & 48,1 & 0,46 & 55,0 & 0,53 & 52,4 & 0,51 & 53,2 & 0,52 \\
\hline $\begin{array}{l}\text { Berufsgattung } \\
\text { (Viersteller) }\end{array}$ & 52,2 & 0,51 & 53,0 & 0,55 & 41,7 & 0,40 & 50,8 & 0,49 & 44,4 & 0,43 & 47,1 & 0,46 \\
\hline
\end{tabular}

Für Angaben zum Beruf des Vaters

\begin{tabular}{|c|c|c|c|c|c|c|c|c|c|c|c|c|}
\hline \multirow[b]{2}{*}{ ISCO-Codierung } & \multicolumn{2}{|c|}{ Vgl_1 } & \multicolumn{2}{|c|}{ Vgl_2 } & \multicolumn{2}{|c|}{ Vgl_3 } & \multicolumn{2}{|c|}{ Vgl_4 } & \multicolumn{2}{|c|}{ Vgl_5 } & \multicolumn{2}{|c|}{ Vgl_6 } \\
\hline & $\overline{\mathrm{PÜ}}$ & $\kappa$ & PÜ & $\kappa$ & PÜ & $\kappa$ & PÜ & $\kappa$ & PÜ & $\kappa$ & PÜ & $\kappa$ \\
\hline $\begin{array}{l}\text { Berufshauptgruppe } \\
\text { (Einsteller) }\end{array}$ & 74,7 & 0,68 & 76,2 & 0,70 & 72,9 & 0,65 & 73,3 & 0,67 & 73,6 & 0,67 & 74,7 & 0,68 \\
\hline $\begin{array}{l}\text { Berufsgruppe } \\
\text { (Zweisteller) }\end{array}$ & 66,0 & 0,63 & 67,3 & 0,65 & 64,2 & 0,61 & 0,62 & 0,60 & 63,2 & 0,60 & 67,6 & 0,65 \\
\hline $\begin{array}{l}\text { Berufsuntergruppe } \\
\text { (Dreisteller) }\end{array}$ & 55,6 & 0,54 & 56,5 & 0,55 & 51,8 & 0,50 & 53,8 & 0,52 & 53,4 & 0,52 & 59,6 & 0,58 \\
\hline $\begin{array}{l}\text { Berufsgattung } \\
\text { (Viersteller) }\end{array}$ & 47,8 & 0,47 & 48,3 & 0,47 & 42,5 & 0,42 & 45,6 & 0,45 & 41,6 & 0,41 & 49,8 & 0,49 \\
\hline
\end{tabular}

Anmerkung: PÜ = Prozentuale Übereinstimmung; $\kappa=$ Kappa.

Vergleiche: Vgl_1 = Institut $1 \&$ Institut 2; Vgl_2 = Institut 1 \& Eigen 1;Vgl_3 = Institut 1 \&

Eigen 2; Vgl_4=Institut $2 \&$ Eigen 1; Vgl_5=Institut $2 \&$ Eigen 2; Vgl_6 = Eigen $1 \&$ Eigen 2.

bei uneindeutigen Angaben zum Beruf hinzugezogen werden und nicht zur Validierung der Codierung insgesamt.

Bezüglich der subjektiven Schwierigkeit, also der Einschätzung, wie sicher sich die Codierer bei der Codierung waren, zeigte sich ein erwarteter Befund. Eine hohe Sicherheitseinschätzung bei der Codierung ging mit einer besseren Übereinstimmung einher. Allerdings korrelierte die subjektiv empfundene Schwierigkeit beim Codieren zwischen den beiden Codierern bei den Angaben der Mutter und des Vaters nur mit $r=0,25$. Demnach gibt es in diesem Punkt durchaus Unterschiede zwischen unterschiedlichen Codierern.

\section{2 Übereinstimmung des sozioökonomischen Status auf Basis unterschiedlicher Berufscodierungen nach der ISCO}

Trotz größerer Diskrepanzen auf der Ebene der ISCO-Codes gingen wir davon aus, dass sich die Werte für den sozioökonomischen Status hinsichtlich der Mittelwerte und Standardabweichungen, die auf Basis unterschiedlicher Codierungen gebildet wurden, 
nicht wesentlich voneinander unterscheiden. Abbildung 2 stellt die Mittelwerte und Standardabweichungen für den ISEI verschiedener Codierungen dar. Insgesamt lassen sich tatsächlich keine größeren Differenzen erkennen. Nach Korrektur des Alpha-Fehlers (nach Bonferroni) ließen sich keine signifikanten Unterschiede zwischen den Mittelwerten des sozioökonomischen Status, die auf Grundlage unterschiedlicher Codierungen gebildet wurden, ermitteln. Dieser Befund besitzt unmittelbare praktische Relevanz für verschiedenste empirische Analysen, denn Unterschiede in den Mittelwerten und Varianzen zwischen verschiedenen Codierungen können insbesondere für deskriptive Analysen bedeutsam werden, wenn Mittelwerte auf Populationsebene miteinander verglichen werden.

Die korrelativen Zusammenhänge des sozioökonomischen Status (ISEI) auf Basis der verschiedenen Codierungen nach der ISCO fielen durchweg hoch aus. Dies lässt sich sowohl für die Werte, die für die Mutter, als auch für die, die für den Vater generiert wurden, feststellen. Mit $r=0,85$ für die Angaben des Vaters und $r=0,86$ für die Angaben der Mutter konnten sehr hohe Korrelationen ermittelt werden. Die Korrelationen auf Basis der Eigencodierung fielen etwas geringer aus, attestieren den unterschiedlichen Codierungen trotzdem einen hohen Zusammenhang ( $r=0,79$ für die Angaben des Vaters und $r=0,74$ für die Angaben der Mutter). In Tab. 2 sind die entsprechenden Korrelationen für die Väter oberhalb und für die Mütter unterhalb der Hauptdiagonalen abgetragen. Es ist davon auszugehen, dass die Korrelationen der Eigencodierung mit den professionellen Codierungen noch höher ausfallen würden, wenn aus der Doppelcodierung ein Wert für

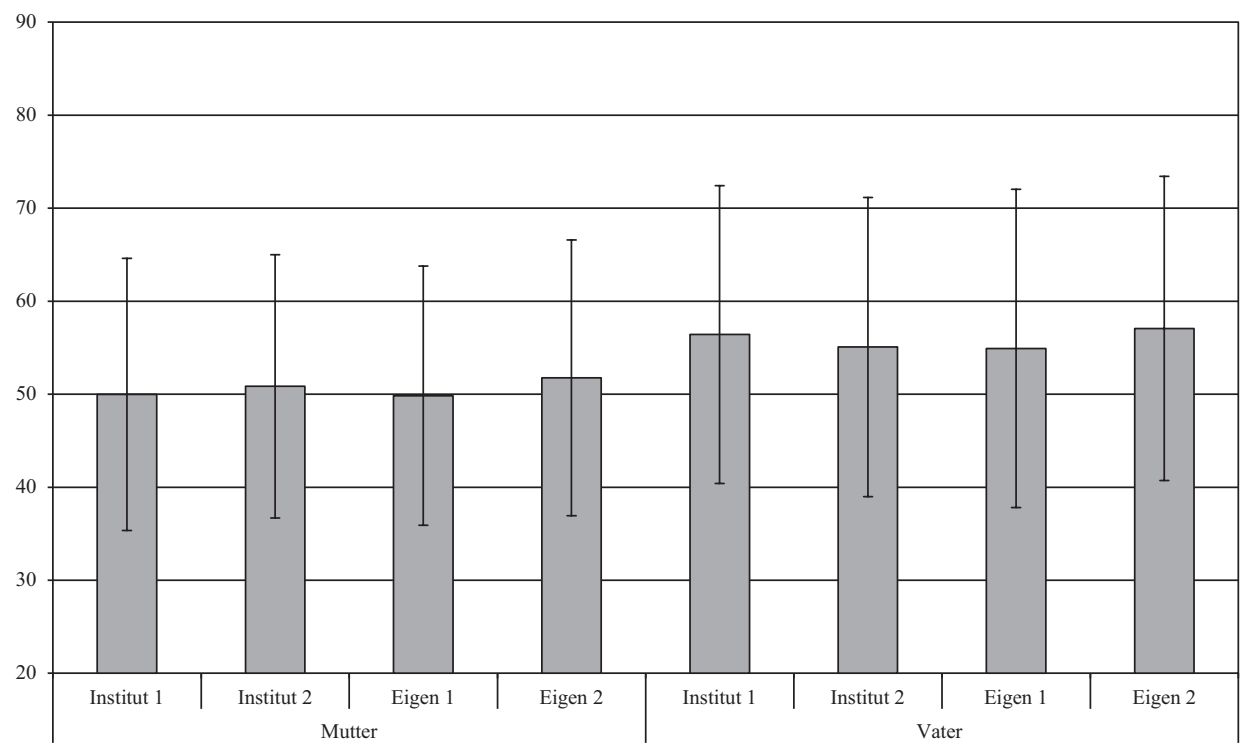

Anmerkung: Institut 1 und 2: ISEI auf Basis der professionellen Codierung durch Fremdinstitute; Eigen 1 und 2: ISEI auf Basis der eigenständigen Codierung durch studentische Mitarbeiter.

Abb. 2: Sozioökonomischer Status der Mütter und Väter, gemessen mit dem International Socio-Economic Index of Occupational Status (ISEI) auf Basis unterschiedlicher Codierungen (Mittelwerte und Standardabweichungen) 
Tab. 2: Korrelationen des sozioökonomischen Status zwischen verschiedenen Codierungen

\begin{tabular}{lllll}
\hline & Institut 1 & Institut 2 & Eigen 1 & Eigen 2 \\
\hline Institut 1 & 1 & 0,85 & 0,81 & 0,80 \\
Institut 2 & 0,86 & 1 & 0,82 & 0,80 \\
Eigen 1 & 0,81 & 0,82 & 1 & 0,79 \\
Eigen 2 & 0,74 & 0,80 & 0,74 & 1 \\
\hline
\end{tabular}

Anmerkung: Angaben für die Väter oberhalb und für die Mütter unterhalb der Hauptdiagonalen; Institut 1 und Institut 2 stehen für die beiden professionellen Institute. Eigen 1 und Eigen 2 stehen für die eigenständig vorgenommenen Codierungen.

die Eigencodierung gebildet würde, aus dem dann der sozioökonomische Status generiert wird.

\subsection{Kriteriumsvalidität des sozioökonomischen Status}

In einer abschließenden Analyse soll die strukturelle Validität unterschiedlicher Berufscodierungen geprüft werden. Hierfür haben wir die Korrelationen zwischen dem sozioökonomischen Status und zentralen Leistungsindikatoren auf der einen Seite und familiären Prozessmerkmalen auf der anderen Seite berechnet. In Tab. 3 sind die Korrelationen zwischen diesen Merkmalen und dem sozioökonomischen Status des Vaters auf Basis unterschiedlicher Berufscodierungen (professionelle und Eigencodierung) dargestellt. Insgesamt zeigt sich ein sehr homogenes Bild, wenngleich Unterschiede zwischen den Codierungen erkennbar sind. Allerdings konnte für keine der berechneten Korrelationen ein statistisch signifikanter Unterschied zwischen den verschiedenen Codierungen ermittelt werden. Für die Werte des sozioökonomischen Status der Mutter zeigte sich ein iden-

Tab. 3: Korrelationen des sozioökonomischen Status (ISEI) für den Vater mit Leistungsindikatoren und familiären Prozessmerkmalen

\begin{tabular}{|c|c|c|c|c|c|c|c|c|}
\hline & \multicolumn{2}{|c|}{ Vater ISEI } & \multirow[b]{2}{*}{$z$-Wert } & \multirow[b]{2}{*}{$p$-Wert } & \multicolumn{2}{|c|}{ Vater ISEI } & \multirow[b]{2}{*}{$z$-Wert } & \multirow[b]{2}{*}{$p$-Wert } \\
\hline & Institut 1 & Institut 2 & & & Eigen 1 & Eigen 2 & & \\
\hline$\overline{\text { Abiturnote }}$ & $-0,14^{*}$ & $-0,15$ & $-0,081$ & 0,468 & $-0,12^{*}$ & $-0,15^{* *}$ & $-0,290$ & 0,386 \\
\hline $\begin{array}{l}\text { Mathematikleis- } \\
\text { tung }\end{array}$ & 0,10 & $0,14^{*}$ & 0,450 & 0,674 & 0,07 & 0,06 & $-0,643$ & 0,260 \\
\hline Englischleistung & 0,09 & 0,07 & $-0,208$ & 0,418 & 0,08 & 0,08 & $-0,382$ & 0,351 \\
\hline $\begin{array}{l}\text { Kognitive } \\
\text { Grundfähigkeit }\end{array}$ & $0,15^{* *}$ & $0,13^{*}$ & $-0,194$ & 0,423 & $0,13^{*}$ & $0,18^{* *}$ & 0,501 & 0,692 \\
\hline $\begin{array}{l}\text { Bücherzahl im } \\
\text { Haushalt }\end{array}$ & $0,37^{* * *}$ & $0,37^{* * *}$ & $-0,009$ & 0,496 & $0,37^{* * *}$ & $0,31^{* * *}$ & $-1,070$ & 0,142 \\
\hline Kulturelle Praxis & $0,13^{*}$ & $0,13^{*}$ & 0,014 & 0,506 & $0,17^{* *}$ & $0,13^{*}$ & $-0,559$ & 0,288 \\
\hline Soziale Praxis & 0,08 & 0,06 & $-0,283$ & 0,389 & 0,10 & 0,03 & $-0,864$ & 0,194 \\
\hline
\end{tabular}
${ }^{*} p<, 05,{ }^{* *} p<, 01,{ }^{* * *} p<, 001$

Anmerkung: Institut 1 und Institut 2 stehen für die beiden professionellen Institute. Eigen 1 und Eigen 2 stehen für die eigenständig vorgenommenen Codierungen. 
Tab. 4a: Regression der Mathematikleistung auf den sozioökonomischen Status und das Vorwissen (standardisierte Regressionskoeffizienten) für unterschiedliche Berufs-codierungen (Fremdcodierung)

\begin{tabular}{lllllrl}
\hline & Institut 1 & \multicolumn{3}{c}{ Institut 2 } & \multicolumn{2}{c}{ Signifikanztest M2 } \\
\hline & M1 & M2 & M1 & M2 & $z$-Wert & $p$-Wert \\
\hline $\begin{array}{l}\text { Sozioökonomischer } \\
\text { Status }\end{array}$ & $0,11^{*}$ & 0,02 & $0,16^{*}$ & 0,07 & 0,699 & 0,758 \\
$\begin{array}{l}\text { Kognitive } \\
\text { Grundfähigkeit }\end{array}$ & & $0,57^{*}$ & & $0,56^{*}$ & $-0,165$ & 0,435 \\
$R^{2}$ & 0,01 & 0,33 & 0,02 & 0,33 & & \\
\hline
\end{tabular}

Anmerkung: sozioökonomischer Status $=\mathrm{HISEI} ;{ }^{*} p<, 05$; M1: bivariates Modell; M2: unter Kontrolle der kognitiven Grundfähigkeit, weitere Kovariaten wurden nicht berücksichtigt.

tisches Befundmuster (tabellarisch nicht ausgewiesen): Es wurde für keine Korrelation ein statistisch signifikanter Unterschied zwischen den beiden Codierern ermittelt.

In der empirischen Bildungsforschung wird aus den Werten des sozioökonomischen Status von Mutter und Vater ein Maß gebildet, das den höchsten Wert in der Familie repräsentiert. Dieser sogenannte HISEI wird dann in den empirischen Analysen als zentraler Indikator für den sozioökonomischen Status der Herkunftsfamilie einer Schülerin bzw. eines Schülers herangezogen. Für die Berechnung sozialer Disparitäten des Kompetenzerwerbs in der PISA-2000-Studie wurde dieser Indikator beispielsweise als Standardindikator der sozialen Herkunft genutzt. In den Tab. $4 \mathrm{a}$ und $4 \mathrm{~b}$ ist die Regression der Mathematikleistung auf den sozioökonomischen Status einmal als bivariates Modell und einmal unter Kontrolle der kognitiven Grundfähigkeiten als einem zentralen Indikator der Vorleistungen der Schülerinnen und Schüler für die verschiedenen Codierungen dargestellt. Die in den Tab. 4a und 4b dargestellten Modelle dienen nicht der detaillierten Analyse der Mathematikleistung, sondern sollen die prädiktive Validität des soziökonomischen Status auf Basis unterschiedlicher Berufscodierungen miteinander vergleichen. In den verschiedenen Modellen zeigten sich keine Unterschiede in der Zusammenhangsstruktur des sozioökonomischen Status mit den Leistungsindikatoren. Für die inhaltliche Analyse macht es demzufolge keinen Unterschied, aus welcher Codierung der sozioökonomische Status generiert wurde.

Tab. 4b: Regression der Mathematikleistung auf den sozioökonomischen Status und das Vorwissen (standardisierte Regressionskoeffizienten) für unterschiedliche Berufscodierungen (Eigencodierung)

\begin{tabular}{lllllll}
\hline & Eigen 1 & \multicolumn{3}{c}{ Eigen 2 } & \multicolumn{3}{c}{ Signifikanztest M2 } \\
\hline & M1 & M2 & M1 & M2 & $z$-Wert & $p$-Wert \\
\hline $\begin{array}{l}\text { Sozioökonomischer } \\
\text { Status }\end{array}$ & $0,14^{*}$ & 0,04 & 0,11 & $-0,01$ & $-0,662$ & 0,254 \\
$\begin{array}{l}\text { Kognitive } \\
\text { Grundfähigkeit }\end{array}$ & & $-0,57^{*}$ & & $0,57^{*}$ & 0,057 & 0,523 \\
$R^{2}$ & 0,02 & 0,33 & 0,01 & 0,33 & & \\
\hline
\end{tabular}

Anmerkung: sozioökonomischer Status $=\mathrm{HISEI} ;{ }^{*} p<, 05 ; \mathrm{M} 1$ : bivariates Modell; M2: unter Kontrolle der kognitiven Grundfähigkeit, weitere Kovariaten wurden nicht berücksichtigt. 


\section{Zusammenfassung und Diskussion}

Im Mittelpunkt der vorliegenden Arbeit stand die Übereinstimmung zentraler Informationen zum sozialen Hintergrund, die auf unterschiedlichen Berufscodierungen beruhen. Angaben zur sozialen Herkunft von Schülerinnen und Schülern markieren in der Bildungsforschung zentrale Indikatoren empirischer Analysen. Aus Angaben der beruflichen Tätigkeit lassen sich Rangskalen und Kategoriensysteme bilden, die in der Bildungsforschung mittlerweile zum Standardrepertoire sozialstatistischer Indikatoren gehören. Die Erfassung ist aber mit verschiedenen Problemen verbunden. Zum einen greift man bei fehlenden Angaben der Eltern oft auf Angaben der Schülerinnen und Schüler zurück und erhält somit Proxy-Angaben zur beruflichen Tätigkeit. Zum anderen setzt die Bildung valider Rangskalen und Kategoriensysteme eine Codierung der Angaben zur beruflichen Tätigkeit voraus, deren Reliabilität oftmals unbekannt ist.

In der vorliegenden Studie, die sich auf Angaben von 300 Abiturienten zum Beruf der Mutter und des Vaters stützte, die durch professionelle Codierer sowie trainierte studentische Hilfskräfte nach der ISCO-88 klassifiziert und in ISEI-Werte transformiert wurden, war die prädiktive Kraft des familiären Hintergrunds unabhängig von den Codierern. Auch die Korrelationen mit anderen Variablen wiesen keinerlei statistisch signifikante Unterschiede zwischen den Codierungen auf. Schließlich wiesen die Mittelwerte für den sozioökonomischen Status, die auf der Basis der vier Codierungen gebildet wurden, keine statistisch signifikanten Unterschiede auf. Insbesondere für die Schulleistungsforschung jüngeren Datums, in der die Mehrzahl der Analysen zum Zusammenhang von familiärem Hintergrund und schulischem Erfolg auf Rangskalen (ISEI, SIOPS) anstatt auf den kategorialen Berufsangaben (z. B. ISCO-88) beruhen, ist dies zunächst ein beruhigender Befund, der als Validitätshinweis der generierten Skalen interpretiert werden kann. Es scheint also insgesamt keine systematischen Unterschiede zwischen den Codierungen durch unterschiedliche (gut trainierte) Personen zu geben, was aber nicht ausschließt, dass bei einer „fehlerfreien“ Messung des sozialen Hintergrunds der Zusammenhang mit den Drittvariablen insgesamt höher ausfallen könnte.

Allerdings fiel die Übereinstimmung auf der Ebene des vierstelligen ISCO-Codes überraschend gering aus. Nur jede zweite Codierung stimmte zwischen den verschiedenen Codierungen exakt überein, bei nur rund drei von vier Codierungen stimmte zumindest die Berufshauptgruppe überein. Diese doch überraschend geringe Übereinstimmung scheint dabei kein studienspezifisches Phänomen zu sein. Auch in der auf Basis der PISA-2003-Schülerdaten für Österreich durchgeführten Studie konnte keine perfekte Übereinstimmung zwischen unterschiedlichen Codierern ermittelt werden, wenngleich die Übereinstimmung insgesamt etwas höher lag als in der vorliegenden Studie (WallnerPaschon 2004).

Wie kommt es zu nicht übereinstimmenden Codierungen und warum wirken sich diese vergleichsweise wenig auf die Rangskalen aus? Die Codierung nach der ISCO sieht insgesamt 390 Codierungen (Berufsgattungen) vor. Die ISCO ist damit eine hoch komplexe Berufsklassifikation mit einem sehr differenziertem Auflösungsniveau. Diese Komplexität wird durch Zuordnung mehrerer Berufe zu einzelnen Berufsgattungen noch erhöht. $\mathrm{Zu}$ nicht-übereinstimmenden Codierungen dürfte u.a. die hierarchische Gliederung der ISCO beitragen. So sind beispielsweise in der Berufshauptgruppe 5 Dienstleistungsbe- 
rufe, Verkäufer in Geschäften und auf Märkten zusammengefasst. Für die Codierung des Berufs Verkäufer scheint damit die Berufshauptgruppe 5 eindeutig. Allerdings werden Verkäufer, die dieser Tätigkeit in einem eigenen Unternehmen nachgehen, nicht der Berufshauptgruppe 5, sondern der Berufshauptgruppe 1 („Angehörige gesetzgebender Körperschaften, leitende Verwaltungsbedienstete und Führungskräfte in der Privatwirtschaft") zugeordnet. Geben Befragte nun als Beruf „Verkäufer“ an, so ist die Codierung mit Unsicherheit behaftet, falls nicht weitere Angaben (z. B. berufliche Tätigkeit, berufliche Stellung, Anzahl der Mitarbeiter) ein eindeutigeres Bild vermitteln. An dieser Stelle wird deutlich, dass die Gründe des Mismatches mit der Erhebung der zu codierenden Merkmale zusammenhängen können. Je umfassender der Beruf erfasst wird, desto mehr Informationen können bei der Codierung herangezogen werden.

Ein weiterer Grund für die hohe Anzahl nicht-übereinstimmender Berufs-Codes nach der ISCO-88 liegt in dem von uns verwendeten strengen Übereinstimmungskriterium. Schwächt man das Kriterium der Übereinstimmung etwas ab und definiert bei gegebenem vierstelligem ISCO-Code auch den korrespondierenden Dreisteller (Berufsuntergruppe) als Übereinstimmung, ist mit einer höheren prozentualen Übereinstimmung zu rechnen.

Unterschiede auf der Ebene der Berufsgattungen wirken sich oftmals nicht oder nur unwesentlich auf die Generierung der Werte des sozioökonomischen Status aus. Das liegt unter anderem daran, dass für eine Reihe von ,ähnlichen“ Berufen, die aber unterschiedlichen Berufsgattungen angehören, die resultierenden ISEI-Werte ähnlich oder gleich ausfallen. Als Beispiele sind Schaffner (ISCO: 5112) und Reiseführer (ISCO: 5113) zu nennen, deren ISEI-Wert jeweils 34 beträgt, sowie Medizinischer Assistent $($ ISCO = 3221) und Pharmazeutischer Assistent (ISCO: 3228), bei denen der ISEI-Wert jeweils 51 beträgt. Der Mismatch ist in diesen Fällen für die empirische Analyse praktisch unbedeutend, was sich entsprechend auch darin ausdrückt, dass - wie oben berichtet - die Zusammenhänge der vier Codierungen mit Drittvariablen vergleichbar ausfielen.

$\mathrm{Da}$ in vielen Forschungsprojekten auf eine professionelle Codierung der Berufe unter anderem aus Kostengründen verzichtet wird, haben wir zusätzlich zur Beauftragung der professionellen Institute eine Eigencodierung von zwei von uns geschulten studentischen Mitarbeiterinnen und Mitarbeitern vorgenommen. Die Übereinstimmung zwischen der Doppelcodierung glich im Wesentlichen dem Vergleich der professionellen Codierungen. Zwar fiel die Übereinstimmung der Eigencodierung mit den professionellen Codierungen geringer aus als die Übereinstimmung der professionellen Codierungen untereinander, erstaunlicherweise liegt sie aber insgesamt sehr dicht an den professionellen Codierungen. Trotzdem fällt es insgesamt schwer, eine Empfehlung zur Verwendung von Eigencodierungen als eine Alternative zur professionellen Codierung abzugeben. Die Güte der Eigencodierung hängt im Wesentlichen von den Vorarbeiten der Codierung ab. Hierunter sind zunächst die Informationen zu zählen, die codiert werden sollen. Die differenzierte Erfassung der beruflichen Tätigkeit scheint ein erster wichtiger Schritt zu sein. In der vorliegenden Studie ist deutlich geworden, dass die einfache Erfassung des elterlichen Berufs keine hinreichende Information für eine valide Codierung darstellt. Darüber hinaus setzt eine Eigencodierung auch „Expertenwissen“ in den jeweiligen Forschungsprojekten voraus, da der Codierung eine entsprechende Schulung der Codierer vorausgehen muss. Für die Eigencodierung wurden den Codierern keine Zeitvorgaben gegeben und in der Tat fiel sie sehr zeitintensiv aus. Berücksichtigt werden muss an dieser Stelle auch die 
verhältnismäßig geringe Zahl der Codierungen (600 Codierungen bei 300 Fällen). Bei größeren Studien (ab 1.000 Probanden) erhöht sich die Zahl der Codierungen schnell auf mehrere Tausend und vervielfacht sich, wenn neben den Berufen der Eltern z. B. auch Ausbildungsberufe und Studienfächer oder Berufsaspirationen codiert werden sollen.

Bezüglich der Nutzung weiterer Informationen zeigte sich, dass die Nutzung vieler Informationen nicht zwingend zu einer besseren Übereinstimmung der Codierungen führt. Dies ist nicht weiter verwunderlich, da die Nutzung vieler Informationen auch darauf hinweisen dürfte, dass die eigentliche Berufsangabe nicht völlig eindeutig war. Uns scheint insbesondere der Hinweis wichtig, dass die differenzierte Erfassung des Berufs in Form der Berufsangabe (z.B. „Verkäufer") sowie - zusätzlich - der genauen Tätigkeit in diesem Beruf (z. B. ,,verkauft Backwaren in einem Geschäft“ ISCO: 5220, „,verkauft Backwaren auf einem Markt" ISCO: 5230) für die Codierung von entscheidender Bedeutung ist. Die doppelte Erfassung von Beruf und Tätigkeit scheint für die genaue Codierung der Berufe unverzichtbar. Welche weiteren Merkmale des Berufs (z. B. Mitarbeiterzahl, berufliche Stellung) besonders wichtig für eine korrekte Codierung sind, sollte in weiteren Studien untersucht werden (Hoffmeyer-Zlotnik, Hess \& Geis 2004).

Zusammenfassend lässt sich konstatieren, dass der in den von uns analysierten Daten vorhandene Messfehler bei den Skalen zur Erfassung des sozioökonomischen Status (ISEI) unsystematischer Natur war. Welche der vier Codierungen herangezogen wurde, war für die substanzwissenschaftlichen Befunde, die sich auf die ISEI-Codierung stützten, unbedeutsam. Dies impliziert jedoch nicht, dass der Messfehler irrelevant ist. So wäre es beispielsweise denkbar, dass die Zusammenhänge zwischen familiärem Hintergrund und schulischen Erfolgsmaßen höher ausfallen würden, wenn es gelingen würde, die Berufe der Eltern messfehlerfrei zu erfassen. Es bedarf deshalb weiterer Analysen, um nach möglichen Quellen für Abweichungen oder nach systematischen Verzerrungen zu suchen. Gerade dem Thema sozialer Disparitäten im Bildungssystem kommt ein großes öffentliches und politisches Interesse zu. Die zuverlässige Messung der Indikatoren, mit denen soziale Disparitäten analysiert werden, ist demnach nicht nur aus wissenschaftlicher Sicht erforderlich, sondern stellt eine Verpflichtung der Wissenschaft gegenüber der Öffentlichkeit und Politik dar. Wir halten deshalb weitere Studien zur Reliabilität der Codierung von Berufsangaben für wünschenswert. Dabei sollte sowohl nach Wegen zur Verbesserung der Codierung vorhandener Angaben als auch nach Möglichkeiten zur Verbesserung der verwendeten Fragebögen gesucht werden.

\section{Anmerkungen}

1 Neben der ISCO-88 wurden regionale Varianten (z.B. für Arbeitsmarkterhebungen in der Europäischen Union) entwickelt, z. B. ISCO-88 (COM), die sich sehr dicht an der ISCO-88 orientieren (Elias \& Birch 1991). Da ISCO-88 und ISCO-88 (COM) auf derselben Logik (der beruflichen Tätigkeit) basieren und nur in einer handvoll Codes voneinander abweichen (vgl. Elias \& Birch 1993), ist Umcodierung von ISCO-88 (COM) in ISCO-88 (und umgekehrt) durch entsprechende Umsteigeschlüssel möglich.

2 URL: http://www.gesis.org/Methodenberatung/Textanalyse/Berufs-Branchenklassifikation/ Beruf_Branche.htm (Download: 06.08.2008) 


\section{Literatur}

Agresti, A. (1990). Categorical data analysis. New York: Wiley \& Sons.

Bauer, A., Langenheim, H. \& Schork, B. (1984). Kinder als Informanten. Eine empirische Untersuchung über die Zuverlässigkeit der Schichteinstufung der Eltern durch Schüler aus der 4. Klasse. In M. Meulemann \& K.-H. Reuband (Hrsg.), Soziale Realität im Interview. Empirische Analysen methodischer Probleme (S. 241-250). Frankfurt am Main: Campus.

Baumert, J. \& Maaz, K. (2006). Das theoretische und methodische Konzept von PISA zur Erfassung sozialer und kultureller Ressourcen der Herkunftsfamilie: internationales und nationales Konzept. In J. Baumert, P. Stanat \& R. Watermann (Hrsg.), Herkunftsbedingte Disparitäten im Bildungswesen: Differenzielle Bildungsprozesse und Probleme der Verteilungsgerechtigkeit (S. 11-29). Wiesbaden: VS-Verlag für Sozialwissenschaften.

Baumert, J., Stanat, P. \& Watermann, R. (Hrsg.) (2006). Herkunftsbedingte Disparitäten im Bildungswesen: Differenzielle Bildungsprozesse und Probleme der Verteilungsgerechtigkeit. Wiesbaden: VS Verlag für Sozialwissenschaften.

Cohen, J. (1960). A coefficient of agreement for nominal scales. Educational and Psychological Measurement, 10, 37-46.

Elias, P. \& Birch, M. (1991). Harmonisierung von Berufsklassifizierungen. ISCO 88 (COM). Vorgeschlagene Fassung der Internationalen Standardklassifikation der Berufe (1988) für nationale Vergleiche der in der Europäischen Gemeinschaft in Volkszählungen und Erhebungen ermittelten Informationen. Warwick: Universität. Zugriff am 11.03.2009 unter www.warwick. ac.uk/ier/isco/GERMISCO.DOC.

Elias, P. \& Birch M. (1993). ISCO 88 (COM). Fassung der Internationalen Standardklassifikation der Berufe 1988 zur Verwendung innerhalb der Europäischen Gemeinschaft. Warwick: Universität. Zugriff am 11.03.2009 unter www.gesis.org/fileadmin/upload/dienstleistung/daten/ amtl_mikrodaten/mz/Panel/ISCO88COM_MZ_1_.pdf.

Educational Testing Service (1997). TOEFL 1997 edition: Test and score manual. Princeton, NJ: Educational Testing Service.

Ganzeboom, H. B. G., Graaf, P. M. de \& Treiman, D. J. (1992). A standard international socio-economic index of occupational status. Social Science Research, 21, 1-56.

Geis, A. (2007). Handbuch für die Berufsvercodung. Mannheim: ZUMA. Zugriff am 11.03.2009 unter http://www.gesis.org/fileadmin/upload/dienstleistung/tools_standards/hdb_0706.pdf.

Geis, A. \& Hoffmeyer-Zlotnik, J. H. P. (2001). Kompatibilität von ISCO-68, ISCO-88 und KldB92. ZUMA-Nachrichten, 25 (48), 117-138.

Heller, K. A. \& Perleth, C. (2000). Kognitiver Fähigkeitstest für 4. bis 12. Klassen, Revision. - Göttingen: Hogrefe.

Hoffmeyer-Zlotnik, J. H. P., Hess, D. \& Geis, A. (2004). Computergestützte Vercodung der International Standard Classification of Occupations (ISCO-88). ZUMA-Nachrichten, 28 (55), $29-52$.

Hoffmeyer-Zlotnik, J. H. P., Hess, D. \& Geis, A. (2006). Computergestützte Vercodung der International Standard Classification of Occupations (ISCO-88). Vorstellen eines Instrumentes. ZUMA-Nachrichten, 30 (58), 101-113.

International Labour Office (Ed.) (1969). International Standard Classification of Occupations. Revised Edition. Geneva: ILO.

International Labour Office (Ed.) (1990). International Standard Classification of Occupations. ISCO-88. Geneva: ILO.

Köller, O. \& Trautwein, U. (2004). Englischleistungen von Schülerinnen und Schülern an allgemein bildenden und beruflichen Gymnasien. In O. Köller, R. Watermann, U. Trautwein \& O. Lüdtke (Hrsg.), Wege zur Hochschulreife in Baden-Württemberg. TOSCA - eine Untersuchung an allgemein bildenden und beruflichen Gymnasien (S. 285-326). Opladen: Leske + Budrich. 
Köller, O., Watermann, R., Trautwein, U. \& Lüdtke, O. (2004). Wege zur Hochschulreife in BadenWürttemberg. TOSCA - eine Untersuchung an allgemein bildenden und beruflichen Gymnasien. Opladen: Leske + Budrich.

Kreuter, F., Maaz, K. \& Watermann, R. (2005). Messung sozialer Herkunft in Schulleistungsuntersuchungen: Der Zusammenhang zwischen der Qualität von Schülerangaben und den Leistungen. In U. Engel (Hrsg.), Bildung und soziale Ungleichheit. Methodologische und strukturelle Analysen (S. 9-35). Bonn: Informationszentrum Sozialwissenschaften.

Kreuter, F., Maaz, K. \& Watermann, R. (2006). Der Zusammenhang zwischen der Qualität von Schülerangaben zur sozialen Herkunft und den Schulleistungen. In K.-S. Rehberg (Hrsg.), Soziale Ungleichheit - Kulturelle Unterschiede. Verhandlungen des 32. Kongresses der Deutschen Gesellschaft für Soziologie in München 2004 (auf CD-ROM: S. 3465-3478). Frankfurt am Main: Campus.

Lien, N., Friestad, C. \& Klepp, K.-I. (2001). Adolescents proxy reports of parents socioeconomic status: How valid are they? Journal of Epidemiology \& Community Health, 55, 731-737.

Maaz, K. \& Watermann, R. (2004). Die Erfassung sozialer Hintergrundmerkmale bei Schülern und Hinweise zu ihrer Validität. In W. Bos, E.-M. Lankes, N. Plaßmeier \& K. Schwippert (Hrsg.), Heterogenität. Eine Herausforderung an die empirische Bildungsforschung (S. 209-229). Münster: Waxmann.

Maaz, K., Kreuter, F. \& Watermann, R. (2006). Schüler als Informanten? Die Qualität von Schülerangaben zum sozialen Hintergrund. In: J. Baumert, P. Stanat \& R. Watermann (Hrsg.), Herkunftsbedingte Disparitäten im Bildungswesen: Differenzielle Bildungsprozesse und Probleme der Verteilungsgerechtigkeit (S. 31-59). Wiesbaden: VS Verlag für Sozialwissenschaften.

Meulemann, H. \& Wieken-Mayser, M. (1984). Kategorien der Sozialstatistik und Alltag der Familie. Die Übereinstimmung von Gymnasiasten des 10. Schuljahres mit ihren Eltern in Angaben und Struktur zur sozialen Position der Familie. In H. Meulemann \& K.-H. Reuband (Hrsg.), Soziale Realität im Interview. Empirische Analysen methodischer Probleme (S. 251-280). Frankfurt am Main: Campus.

Statistisches Bundesamt (Hrsg.) (2004). Demographische Standards. Wiesbaden: DESTATIS.

Trautwein, U., Köller, O., Lehmann, R. \& Lüdtke, O. (2007). Schulleistungen von Abiturienten: Regionale, schulformbezogene und soziale Disparitäten. Münster: Waxmann.

Wallner-Paschon, C. (2004). Berufsklassifizierung. In: C. Reiter, B. Lang \& G. Haider (Hrsg.), PISA 2003. Internationaler Vergleich von Schülerleistungen. Technischer Bericht Mathematik Lese-Kompetenz Naturwissenschaft Problemlösen (S. 161-182). Wien: Bundesministerium für Bildung, Wissenschaft und Kultur.

Watermann, R., Nagy, G. \& Köller, O. (2004). Mathematikleistungen in allgemein bildenden und beruflichen Gymnasien. In O. Köller, R. Watermann, R., U. Trautwein \& O. Lüdtke (Hrsg.), Wege zur Hochschulreife in Baden-Württemberg. TOSCA - eine Untersuchung an allgemein bildenden und beruflichen Gymnasien (S. 205-283). Opladen: Leske + Budrich.

Wirtz, M. \& Caspar, F. (2002). Beurteilerübereinstimmung und Beurteilerreliabilität. Methoden zur Bestimmung und Verbesserung der Zuverlässigkeit von Einschätzungen mittels Kategoriensystemen und Ratingskalen. Göttingen: Hogrefe. 